\title{
REPORT 03-09
}

ON THE FREE VIBRATIONS OF A RECTANGULAR PLATE WITH TWO OPPOSITE SIDES SIMPLY SUPPORTED AND THE OTHER SIDES ATTACHED TO LINEAR SPRINGS.

M.A. Zarubinskaya W.T. van Horssen.

ISSN $1389-6520$

Reports of the Department of Applied Mathematical Analysis

Delft 2003 
Copyright (C 2000 by Department of Applied Mathematical Analysis, Delft, The Netherlands.

No part of the Journal may be reproduced, stored in a retrieval system, or transmitted, in any form or by any means, electronic, mechanical, photocopying, recording, or otherwise, without the prior written permission from Department of Applied Mathematical Analysis, Delft University of Technology, The Netherlands. 


\title{
On the free vibrations of a rectangular plate with two opposite sides simply supported and the other sides attached to linear springs.
}

\author{
M.A. Zarubinskaya and W.T. van Horssen
}

\begin{abstract}
In this paper an initial-boundary value problem for a plate equation will be studied. This initialboundary value problem can be regarded as a rather simple model describing free oscillations of a suspension bridge. The suspension bridge is modeled as a rectangular plate with two opposite sides simply supported and the other sides attached to linear springs. An adapted version of the method of separation of variables is used to find the eigenfrequencies for this plate configuration.
\end{abstract}

\section{Introduction}

Plates of various geometries, i.e. circular, annular, rectangular, polygonal, etc, and of orthotropic material are extensively used in engineering applications. These plates are widely used in modern aerospace technology, naval structural engineering, aircraft structures, and so on. A lot of literature exists for the free vibrations of rectangular plates. In most of these papers the classical theory for isotropic, homogeneous, thin plates with uniform thickness is used and the differential equation to describe the vibrations of the plate is given by

$$
D\left(u_{x x x x}+2 u_{x x y y}+u_{y y y y}\right)+\rho \frac{\partial^{2} u}{\partial t^{2}}=0,
$$

where $D=E h^{3} / 12\left(1-\nu^{2}\right)$ is the flexural rigidity, $E$ is Young's modulus, $\nu$ is Poisson's ratio with $0<\nu<1, \rho$ is the mass density per unit area of the plate surface, $h$ is the thickness of the plate, $t$ is time, and $u(x, y, t)$ is the displacement of the plate in the $z$-direction. The majority of literature deals with classical boundary conditions representing clamped, simply supported, or free edges, and only a small number deals with edges which are restrained against translation or/and rotation, or with other nonclassical boundary conditions. It has been observed for rectangular plates by Leissa in [1]-[3] that there exist 21 distinct cases which involve all possible combinations of classical boundary conditions. For six cases having two opposite sides simply-support it is well-known that exact solutions exist which are in fact the extensions of Voight's work. In [2] Leissa gives a survey of research on rectangular plate problems up to 1970. For a further overview up to beginning of this century the reader is referred to [4]-[9].

One of the most commonly used methods in free vibration analysis of plates is the Rayleigh-Ritz energy technique, where appropriate functions associated with various boundary conditions are chosen to describe the lateral deflection of the deformed plates. The chosen functions almost always do not satisfy the governing differential equation. Also, the functions may or may not satisfy all of the boundary conditions. Thus, the results obtained by the Rayleigh-Ritz method are approximate. Gorman in [4] and [5] succeeded in solving approximately free vibration problems of plates for various geometries and boundary conditions. Compared to the Rayleigh-Ritz method, the superposition technique in [4] and [5] allows one to obtain an analytical form of the solution which satisfies the governing differential equation and the boundary conditions. Sakata and Hosokawa [6] studied the forced and free vibration of clamped orthotropic plates by using a double trigonometric series. During the last forty years the free vibrations of rectangular plates were studied intensively (see for instance [4]-[9], and the references in those papers). In this paper the free vibrations of a rectangular plate with two opposite edges simply supported, and 
linear springs densely attached to the two other edges will be studied. This boundary support will lead to boundary conditions which seem to be not studied in the existing literature.

Flexible structures, like tall buildings and suspension bridges are subjected to oscillations due to windforces or other various causes. Simple models which describe these oscillations are given in the form of weakly nonlinear second- and fourth-order partial differential equations, as can be seen in [10]- [16]. Usually asymptotic methods can be used to construct approximations for the solutions of these wave or beam equations. In [16] a survey of the literature on oscillations in suspension bridges is given. A simple way to model the behaviour of a suspension bridge is to describe it as a vibrating one-dimensional beam with simply supported ends. In [16] the other two dimensions are not taken into account because the dimensions of the bridge in these directions are assumed to be small compared to the length of the bridge. When the width of the bridge is taken into account a plate equation like (1) is obtained. To study for instance wind-induced oscillations of suspension bridges one can of course use plate equations to describe the displacements of the deck of the bridge. However, to investigate these weakly nonlinear wind-induced vibrations we first have to know the related linear vibrations of the rectangular plate with the boundary conditions as described before and as indicated in Figure 1. For that reason we will study in this paper these linear vibrations. Using the results as obtained in this paper one can start to investigate the weakly nonlinear vibrations of a plate in a windfield as model for the wind-induced oscillations of a suspension bridge.

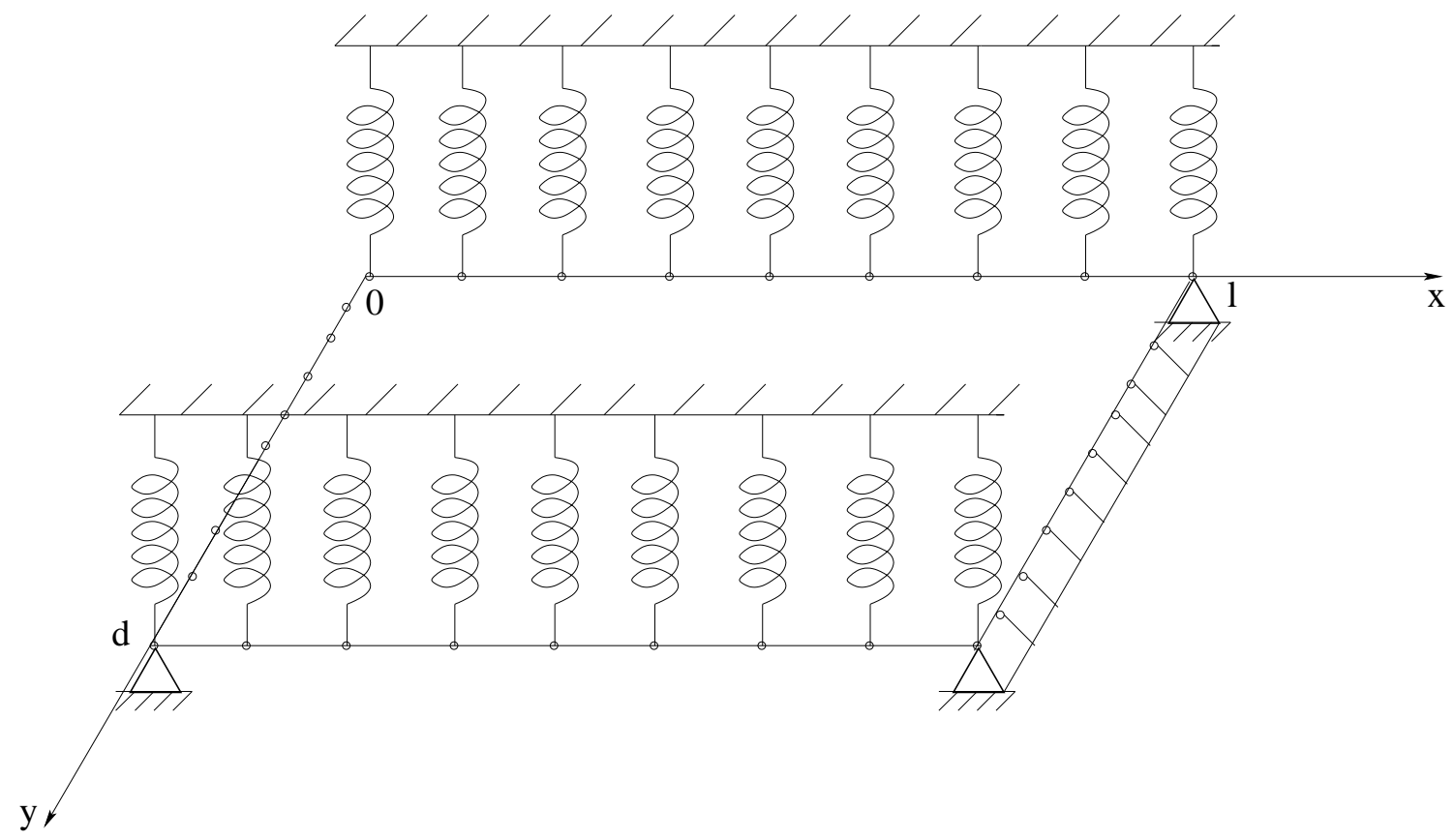

Figure 1. A model of a suspension bridge. 


\section{The mathematical analysis of the problem.}

In this section the following initial-boundary value problem for the displacement function $u(x, y, t)$ will be considered

$$
\begin{aligned}
u_{t t}+D_{1}\left(u_{x x x x}+2 u_{x x y y}+u_{y y y y}\right)=0, & 0<x<l, 0<y<d, t>0, \\
u(x, y, 0)=u_{0}(x, y), \quad u_{t}(x, y, 0)=u_{1}(x, y), & 0<x<l, 0<y<d, \\
u(0, y, t)=u(l, y, t)=u_{x x}(0, y, t)=u_{x x}(l, y, t)=0, & 0<y<d, \\
D\left(u_{y y y}+(2-\nu) u_{x x y}\right)=-p^{2} u, & \text { for } y=0,0<x<l \\
D\left(u_{y y y}+(2-\nu) u_{x x y}\right)=p^{2} u, & \text { for } y=d, 0<x<l \\
u_{y y}+\nu u_{x x}=0, & \text { for } y=0, y=d, 0<x<l,
\end{aligned}
$$

where $D_{1}=\frac{D}{\rho}$, and where $p^{2}$ represents the linear restoring force of the springs (see also Figure 1). The initial displacement and the initial velocity of the plate in $z$-direction are given by $u_{0}(x, y)$ and $u_{1}(x, y)$ respectively. The method of separation of variables will be used to find nontrivial solutions of the boundary value problem (2), (4)-(7), that is, nontrivial solutions in the form

$$
T(t) v(x, y)
$$

will be constructed for the boundary value problem (2), (4)-(7). By substituting (8) into (2) and by dividing the so-obtained equation by $T(t) v(x, y)$, it is follows that

$$
\frac{T^{\prime \prime}}{T}+D_{1} \frac{v_{x x x x}+2 v_{x x y y}+v_{y y y y}}{v}=0 .
$$

From this equation the following two ODEs are obtained

$$
\begin{aligned}
& \frac{T^{\prime \prime}}{T}=-\alpha D_{1}, \\
& v_{x x x x}+2 v_{x x y y}+v_{y y y y}=\alpha v,
\end{aligned}
$$

where $\alpha \in \mathbb{C}$ is a separation parameter. From the boundary conditions (4)-(7) it follows that $v$ has to satisfy

$$
\begin{gathered}
v(0, y)=v(l, y)=v_{x x}(0, y)=v_{x x}(l, y)=0, \quad 0<y<d, \\
D\left(v_{y y y}+(2-\nu) v_{x x y}\right)=-p^{2} v, \quad \text { for } y=0,0<x<l, \\
D\left(v_{y y y}+(2-\nu) v_{x x y}\right)=p^{2} v, \text { for } y=d, 0<x<l, \\
v_{y y}+\nu v_{x x}=0 \quad \text { for } y=0, \text { and } y=d, 0<x<l .
\end{gathered}
$$

First it will be shown that the nontrivial solutions of the boundary value problem (10)-(14), that is, the eigenfunctions of (10)-(14) are mutually orthogonal on $0<x<l$ and $0<y<d$. Let $v_{1}(x, y)$ and $v_{2}(x, y)$ be two different eigenfunctions belonging to the different eigenvalues $\alpha_{1}$ and $\alpha_{2}$ respectively. Thus

$$
\begin{aligned}
& v_{1 x x x x}+2 v_{1 x x y y}+v_{1 y y y y}=\alpha_{1} v_{1}, \\
& v_{2 x x x x}+2 v_{2 x x y y}+v_{2 y y y y}=\alpha_{2} v_{2},
\end{aligned}
$$

where both functions $v_{1}$ and $v_{2}$ satisfy the boundary conditions (11)-(14). It wil be shown that

$$
\left(\alpha_{2}-\alpha_{1}\right) \int_{0}^{d} \int_{0}^{l} v_{1} v_{2} d x d y=0 .
$$

Let the differential operator $A$ be given by

$$
A=\frac{\partial^{4}}{\partial x^{4}}+2 \frac{\partial^{4}}{\partial x^{2} \partial y^{2}}+\frac{\partial^{4}}{\partial y^{4}},
$$


and consider

$$
\int_{0}^{d} \int_{0}^{l}\left(v_{1} A v_{2}-v_{2} A v_{1}\right) d x d y
$$

By using (15) it follows that

$$
\int_{0}^{d} \int_{0}^{l}\left(v_{1} A v_{2}-v_{2} A v_{1}\right) d x d y=\left(\alpha_{2}-\alpha_{1}\right) \int_{0}^{d} \int_{0}^{l} v_{1} v_{2} d x d y
$$

On the other hand by integrating by parts two times it follows that

$$
\begin{aligned}
\int_{0}^{d} \int_{0}^{l}\left(v_{1} A v_{2}-v_{2} A v_{1}\right) d x d y & =\int_{0}^{d} \int_{0}^{l}\left(v_{1 x x} v_{2 x x}+2 v_{1 x y} v_{2 x y}+v_{1 y y} v_{2 y y}\right) d x d y \\
& +\left.\int_{0}^{l}\left(2 v_{1} v_{2 x x y}+v_{1} v_{2 y y y}-v_{1 y} v_{2 y y}\right)\right|_{y=0} ^{d} d x \\
& -\int_{0}^{d} \int_{0}^{l}\left(v_{1 x x} v_{2 x x}+2 v_{1 x y} v_{2 x y}+v_{1 y y} v_{2 y y}\right) d x d y \\
& -\left.\int_{0}^{l}\left(2 v_{2} v_{1 x x y}+v_{2} v_{1 y y y}-v_{2 y} v_{1 y y}\right)\right|_{y=0} ^{d} d x .
\end{aligned}
$$

Using the boundary conditions (14) it follows that the integral

$$
\left.\int_{0}^{l}\left(v_{2 y} v_{1 y y}-v_{1 y} v_{2 y y}\right)\right|_{y=0} ^{d} d x=-\left.\int_{0}^{l} \nu\left(v_{2 y} v_{1 x x}-v_{1 y} v_{2 x x}\right)\right|_{y=0} ^{d} d x .
$$

Integrating the integral two times by parts, and by using the boundary conditions (11)-(14) it follows that

$$
\left.\int_{0}^{l}\left(v_{2 y} v_{1 y y}-v_{1 y} v_{2 y y}\right)\right|_{y=0} ^{d} d x=\left.\int_{0}^{l} \nu\left(v_{2} v_{1 x x y}-v_{1} v_{2 x x y}\right)\right|_{y=0} ^{d} d x
$$

After substituting the last expression and the boundary conditions (12), and (13) into (20), it finally follows that

$$
\int_{0}^{d} \int_{0}^{l}\left(v_{1} A v_{2}-v_{2} A v_{1}\right) d x d y=0
$$

From (19) and (21) it follows that (16) is true, and therefore $v_{1}$ and $v_{2}$ are orthogonal for $\alpha_{1} \neq \alpha_{2}$.

Now it will be shown that the eigenvalue $\alpha$ is real. Let $v(x, y)$ be an eigenfunction belonging to the eigenvalue $\alpha$, so $A v=\alpha v$. Consider $\overline{A v}=\overline{\alpha v}$. Then, replacing in (19) and (21) the functions $v_{1}$ and $v_{2}$ by $v$ and $\bar{v}$ respectively, and using the fact that $A v=\alpha v$ and $A \bar{v}=\bar{\alpha} \bar{v}$ in (19) and (21) it similarly follows that

$$
(\alpha-\bar{\alpha}) \int_{0}^{d} \int_{0}^{l} v \bar{v} d x d y=0
$$


Since $v$ and $\bar{v}$ are eigenfunctions it follows that $\int_{0}^{d} \int_{0}^{l} v \bar{v} d x d y>0$, and so it follows from (22) that $\alpha-\bar{\alpha}=0$. It also can be shown elementarily that $\alpha>0$ by considering $\int_{0}^{d} \int_{0}^{l} v A v d x d y$, where $v(x, y)$ is an eigenfunction belonging to the eigenvalue $\alpha$, that is, $A v=\alpha v$. Firstly it should be observed that

$$
\int_{0}^{d} \int_{0}^{l} v A v d x d y=\alpha \int_{0}^{d} \int_{0}^{l} v^{2}(x, y) d x d y
$$

and secondly it follows (by integrating by parts, and by using the boundary conditions (11)-(14)) that

$$
\begin{aligned}
\int_{0}^{d} \int_{0}^{l} v A v d x d y & =\int_{0}^{d} \int_{0}^{l} v\left(v_{x x x x}+2 v_{x x y y}+v_{y y y y}\right) d x d y \\
& =\int_{0}^{d} \int_{0}^{l}\left(v_{x x}^{2}+2 v_{x y}^{2}+v_{y y}^{2}\right) d x d y+\bar{p}^{2} \int_{0}^{l}\left(v^{2}(d)+v^{2}(0)\right) d x
\end{aligned}
$$

where $\bar{p}^{2}=\frac{p^{2}}{D}$. From (23) and (24) it can readily be deduced that $\alpha>0$.

To investigate the boundary-value problem (10)-(14) for $v(x, y)$ further the method of separation of variables will be used again, that is, it is assumed that a nontrivial solution of the boundary-value problem (10)-(14) can be found in the form

$$
X(x) Y(y)
$$

By substituting (25) into (10) it follows that

$$
\frac{\dddot{X}}{X}+2 \frac{\ddot{X}}{X} \frac{Y^{\prime \prime}}{Y}+\frac{Y^{\prime \prime \prime \prime}}{Y}=\alpha,
$$

where $^{\prime}=\frac{\partial(\ldots)}{\partial y}$ and ${ }^{\prime}=\frac{\partial(\ldots)}{\partial x}$. Generally it is assumed that the variables in (26) can not be separated because of the mixed term $2 \frac{\ddot{X}}{X} \frac{Y^{\prime \prime}}{Y}$. However, using an adapted version of the method of separation of variables (see [17], and [18]), this equation can easily be separated by simply differentiating (26) with respect to $x$ or $y$. For instance, if $(26)$ is differentiated with respect to $\mathrm{x}$ it follows that

$$
\frac{d}{d x}\left(\frac{\dddot{X}}{X}\right)+2 \frac{Y^{\prime \prime}}{Y} \frac{d}{d x}\left(\frac{\ddot{X}}{X}\right)=0,
$$

and so,

$$
\frac{Y^{\prime \prime}}{Y}=-\gamma
$$

where $\gamma \in \mathbb{C}$ is a separation parameter. From (27) it follows that $Y^{\prime \prime \prime \prime}=-\gamma Y^{\prime \prime}=\gamma^{2} Y$, and then it can be deduced from $(26)$ that $X(x)$ and $Y(y)$ have to satisfy

$$
\begin{aligned}
& \dddot{X}-2 \gamma \ddot{X}+\left(\gamma^{2}-\alpha\right) X=0, \quad 0<x<l, \\
& Y^{\prime \prime}=-\gamma Y, \quad 0<y<d .
\end{aligned}
$$

By substituting (25) into the boundary conditions (11)-(14) the usual boundary-value problem for $X(x)$ and for $Y(y)$ are obtained. It turns out, however, that these boundary value problems only admit the trivial solution. The elementary calculations to obtain this result will be omitted.

So, differentiation with respect to $x$ leads only to the trivial solution. However, if (26) is differentiated with respect to $y$ it will turn out that nontrivial solutions can be found. When (26) is differentiated with respect to $y$ it follows that

$$
2 \frac{\ddot{X}}{X} \frac{d}{d y}\left(\frac{Y^{\prime \prime}}{Y}\right)+\frac{d}{d y}\left(\frac{Y^{\prime \prime \prime \prime}}{Y}\right)=0
$$


which can be easily separated, yielding

$$
\frac{\ddot{X}}{X}=-\beta
$$

where $\beta \in \mathbb{C}$ is a separation parameter. From (30) it follows that $\dddot{X}=-\beta \ddot{X}=\beta^{2} X$, and then it can be deduced from (26) and the boundary conditions (12)-(14) that $Y(y)$ has to satisfy

$$
Y^{\prime \prime \prime \prime}-2 \beta Y^{\prime \prime}+\left(\beta^{2}-\alpha\right) Y=0
$$

subject to the boundary conditions

$$
\begin{aligned}
D\left(Y^{\prime \prime \prime}-(2-\nu) \beta Y^{\prime}\right)=-p^{2} Y & \text { for } \quad y=0, \\
D\left(Y^{\prime \prime \prime}-(2-\nu) \beta Y^{\prime}\right)=p^{2} Y & \text { for } \quad y=d, \\
Y^{\prime \prime}-\beta \nu Y=0 & \text { for } \quad y=0, d .
\end{aligned}
$$

It follows from (11) that $X(x)$ also has to satisfy

$$
X(0)=X(l)=\ddot{X}(0)=\ddot{X}(l)=0 .
$$

The non-trivial solutions of the differential equation (30) subject to the boundary conditions (35) are given by

$$
X(x)=\gamma_{n} \sin \left(\sqrt{\beta_{n}} x\right), \quad \beta_{n}=\left(\frac{n \pi}{l}\right)^{2}
$$

with $n \in \mathbb{Z}^{+}$, and where $\gamma_{n}$ is an arbitary constant. The characteristic equation for the ODE (31) now becomes:

$$
k^{4}-2 \beta_{n} k^{2}+\beta_{n}^{2}-\alpha=0 \Longleftrightarrow\left(k^{2}-\beta_{n}\right)^{2}=\alpha .
$$

In this section it already has been shown that $\alpha>0$. So, only the following three cases have to be considered in (37)

$$
\alpha>\beta_{n}^{2}, \quad 0<\alpha<\beta_{n}^{2}, \quad \text { and } \quad \alpha=\beta_{n}^{2} .
$$

\subsection{The case $\alpha>\beta_{n}^{2}$.}

The solutions of the characteristic equation (37) in this case will be

$$
\sqrt{\sqrt{\alpha}+\beta_{n}}, \quad-\sqrt{\sqrt{\alpha}+\beta_{n}}, \quad i \sqrt{\sqrt{\alpha}-\beta_{n}}, \quad \text { and }-i \sqrt{\sqrt{\alpha}-\beta_{n}}
$$

and the solution of the differential equation (31) can be written in the form

$$
Y(y)=C_{1} \cosh \left(\sqrt{\sqrt{\alpha}+\beta_{n}} y\right)+C_{2} \sinh \left(\sqrt{\sqrt{\alpha}+\beta_{n}} y\right)+C_{3} \cos \left(\sqrt{\sqrt{\alpha}-\beta_{n}} y\right)+C_{4} \sin \left(\sqrt{\sqrt{\alpha}-\beta_{n}} y\right),
$$

where $C_{1}, C_{2}, C_{3}$, and $C_{4}$ are constants of integration.

By substituting (38) into the four boundary conditions (32)-(34) a system of four equations for $C_{1}$, $C_{2}, C_{3}$, and $C_{4}$ is obtained. To find nontrivial solutions for $Y(y)$ the determinant of the corresponding coeffitient matrix should be set equal to zero, that is,

$$
\left|\begin{array}{cccc}
a^{2}-\nu \beta_{n} & 0 & -\left(r^{2}+\nu \beta_{n}\right) & 0 \\
\left(a^{2}-\nu \beta_{n}\right) \cosh (a d) & \left(a^{2}-\nu \beta_{n}\right) \sinh (a d) & -\left(r^{2}+\nu \beta_{n}\right) \cos (r d) & -\left(r^{2}+\nu \beta_{n}\right) \sin (r d) \\
\bar{p}^{2} & a_{1} & -r_{1} \\
a_{1} \sinh (a d)-\bar{p}^{2} \cosh (a d) & a_{1} \cosh (a d)-\bar{p}^{2} \sinh (a d) & r_{1} \sin (r d)-\bar{p}^{2} \cos (r d) & -r_{1} \cos (r d)-\bar{p}^{2} \sin (r d)
\end{array}\right|=0,
$$

where $a=\sqrt{\sqrt{\alpha}+\beta_{n}}, r=\sqrt{\sqrt{\alpha}-\beta_{n}}, a_{1}=a\left(a^{2}-(2-\nu) \beta_{n}\right)$ and $r_{1}=r\left(r^{2}+(2-\nu) \beta_{n}\right)$. From (39) the eigenvalues $\alpha$ can be calculated. The eigenvalues $\alpha$ depend on the parameters $n, \bar{p}^{2}, \nu$, the length $l$, and the width $d$ of the rectangular plate. When the parameter $\bar{p}^{2}$ tends to zero, the boundary conditions correspond to the case for the plate with two opposite edges simply supported and the other two free. When the parameter $\bar{p}^{2}$ tends to infinity the boundary conditions correspond to the case for a plate with all edges simply supported.

We calculate numerically form (39) some eigenvalues $\alpha$ for some values of the parameters. Some of the numerical approximations for $\alpha$ up to 50000 are given in Table 1 and Table 2 . 
Table 1. Approximations of the eigenvalues $\alpha$.

\begin{tabular}{|c|c|c|c|c|c|c|}
\hline $\mathrm{n}$ & \multicolumn{6}{|c|}{$\nu=0.3, l=10, d=1, \bar{p}^{2}=1$} \\
\hline 1 & 1.9861 & 7.6513 & 516.1518 & 3829.3326 & 14657.3213 & 40001.3080 \\
\hline 2 & 2.1484 & 12.7612 & 538.9518 & 3882.7911 & 14752.4921 & 40149.9413 \\
\hline 3 & 2.7739 & 21.6547 & 577.3297 & 3972.2310 & 14911.4787 & 40398.0399 \\
\hline 4 & 4.4052 & 34.8982 & 631.8441 & 4098.1669 & 15134.8325 & 40746.1682 \\
\hline 5 & 7.8156 & 53.2853 & 703.2675 & 4261.3209 & 15423.3239 & 41195.1144 \\
\hline 6 & 14.0147 & 77.8385 & 792.5844 & 4462.6248 & 15777.9403 & 41745.8900 \\
\hline 7 & 24.2497 & 109.8092 & 900.9942 & 4703.2228 & 16199.8847 & 42399.7278 \\
\hline 8 & 40.0047 & 150.6790 & 1029.9158 & 4984.4739 & 16690.5743 & 43158.0810 \\
\hline 9 & & 202.1601 & 1180.9932 & 5307.9560 & 17251.6399 & 44022.6214 \\
\hline \multirow[t]{2}{*}{10} & & 266.1959 & 1356.1002 & 5675.4693 & 17884.9256 & 44995.2379 \\
\hline & \multicolumn{6}{|c|}{$\nu=0.43, l=10, d=1, \bar{p}^{2}=1$} \\
\hline 1 & 1.9893 & 7.3471 & 515.1976 & 3827.7181 & 14655.0633 & 39998.4052 \\
\hline 2 & 2.1504 & 11.5408 & 535.1470 & 3876.7395 & 14743.4633 & 40138.3321 \\
\hline 3 & 2.7397 & 18.8953 & 568.8103 & 3957.7389 & 14891.1757 & 40371.9266 \\
\hline 4 & 4.2548 & 29.9595 & 616.7939 & 4072.4616 & 15098.7686 & 40699.7629 \\
\hline 5 & 7.4164 & 45.4983 & 679.9250 & 4221.2733 & 15367.0360 & 41122.6440 \\
\hline 6 & 13.1786 & 66.5027 & 759.2433 & 4405.1593 & 15696.9969 & 41641.6012 \\
\hline 7 & & 94.1809 & 855.9993 & 4625.3260 & 16089.8944 & 42257.8931 \\
\hline 8 & & 129.9639 & 971.6561 & 4883.2029 & 16547.1949 & 42973.0051 \\
\hline 9 & & 175.5054 & 1067.8944 & 5180.4432 & 17070.5871 & 43788.6480 \\
\hline \multirow[t]{2}{*}{10} & & 232.6826 & 1266.6159 & 5518.9257 & 17661.9817 & 44706.7578 \\
\hline & \multicolumn{6}{|c|}{$\nu=0.5, l=10, d=1, \bar{p}^{2}=1$} \\
\hline 1 & 1.9909 & 7.1830 & 514.6837 & 3826.8486 & 14653.8474 & 8422 \\
\hline 2 & 2.1494 & 10.8793 & 533.0951 & 3872.8643 & 14738.6010 & 40132.0807 \\
\hline 3 & 2.7106 & 17.3876 & 564.2076 & 3949.9290 & 14880.2403 & 7.8637 \\
\hline 4 & 4.1396 & 27.2307 & 608.6426 & 4058.6003 & 15079.3394 & 40674.7693 \\
\hline 5 & 7.1170 & 41.1420 & 667.2432 & 4199.6589 & 15336.7011 & 41083.6059 \\
\hline 6 & & 60.0667 & 741.0620 & 4374.1082 & 15653.3555 & 41585.4120 \\
\hline 7 & & 85.1638 & 831.3573 & 4583.1744 & 16030.5590 & 42181.4549 \\
\hline 8 & & 117.8072 & 939.5938 & 4828.3068 & 16649.7934 & 42873.2305 \\
\hline 9 & & 159.5872 & 1067.4469 & 5111.1785 & 16972.7648 & 43662.4623 \\
\hline \multirow[t]{2}{*}{10} & & 212.3121 & 1216.8074 & 5433.6866 & 17541.4029 & 44551.3210 \\
\hline & \multicolumn{6}{|c|}{$\nu=0.4, l=10, d=1, \bar{p}^{2}=10$} \\
\hline 1 & 17.2169 & 60.0325 & 589.4929 & 3901.0656 & 14728.0124 & 40071.2956 \\
\hline 2 & 17.7689 & 64.4837 & 609.6992 & 3950.7358 & 14817 & 2310 \\
\hline 3 & 18.9723 & 72.2716 & 643.8309 & 4033.8802 & 14968.2555 & 40450.1715 \\
\hline 4 & 21.2800 & 83.9501 & 692.5434 & 4151.0405 & 15179.4562 & 40782.6888 \\
\hline 5 & 25.3670 & 100.2956 & 756.7155 & 4302.9762 & 15452.3532 & 41211.5827 \\
\hline 6 & 32.1501 & 122.3071 & 837.4325 & 4490.6657 & 15787.9589 & 41737.8801 \\
\hline 7 & 42.7963 & 151.2070 & 935.9796 & 4715.3078 & 16187.5082 & 42362.8341 \\
\hline 8 & 58.7241 & 188.4419 & 1053.8436 & 4978.3231 & 16652.4577 & 43087.9233 \\
\hline 9 & 81.5997 & 235.6833 & 1192.7194 & 5281.3557 & 17184.4577 & 43914.8507 \\
\hline \multirow[t]{2}{*}{10} & 113.3323 & 294.8280 & 1354.5169 & 5626.2753 & 17785.4892 & 44845.5433 \\
\hline & \multicolumn{6}{|c|}{$\nu=0.46, l=10, d=1, \bar{p}^{2}=100$} \\
\hline 1 & 69.6196 & 475.0370 & 1368.5399 & 4705.0375 & 15489.0254 & 40811.9114 \\
\hline 2 & 73.0771 & 480.9335 & 1385.0898 & 4750.7959 & 15575.3462 & 40949.6487 \\
\hline 3 & 79.0445 & 491.0580 & 1413.1931 & 4827.4891 & 15719.6021 & 41179.5956 \\
\hline 4 & 87.8447 & 505.8623 & 1453.6163 & 4935.7557 & 15922.3743 & 41502.3286 \\
\hline 5 & 99.9592 & 525.9896 & 1507.4054 & 5076.4798 & 16184.4744 & 41918.6541 \\
\hline 6 & 116.0529 & 552.2844 & 1575.8641 & 5250.7839 & 16506.9433 & 42429.6078 \\
\hline
\end{tabular}


Extension of Table 1.

\begin{tabular}{|r|c|c|c|c|c|c|}
\hline & \multicolumn{7}{|c|}{$\nu=0.46, l=10, d=1, \bar{p}^{2}=100$} \\
\hline 7 & 137.0004 & 585.8012 & 1660.5304 & 5460.0203 & 16891.0493 & 43036.4538 \\
8 & 163.9098 & 627.8138 & 1763.1549 & 5705.7649 & 17338.2866 & 43740.6841 \\
9 & 198.1431 & 679.8229 & 1885.6846 & 5989.8110 & 17850.3741 & 44544.0172 \\
10 & 241.3291 & 743.5613 & 2030.2517 & 6314.1662 & 18429.2540 & 45448.3979 \\
\hline
\end{tabular}

Table 2. Approximations of the eigenvalues $\alpha$ for $\nu=0.3, l=10$.

\begin{tabular}{|r|l|l|l|l|}
\hline $\mathrm{n}$ & \multicolumn{2}{|c|}{$d=0.1, \bar{p}^{2}=1$} & \multicolumn{2}{c|}{$d=0.1, \bar{p}^{2}=10$} \\
\hline 1 & 20.0095 & 225.8181 & 199.9985 & 765.8022 \\
2 & 20.1454 & 723.3859 & 200.1480 & 1263.3647 \\
3 & 20.7266 & 1553.0419 & 200.7735 & 2093.0118 \\
4 & 22.2851 & 2715.3508 & 202.3940 & 3255.3083 \\
5 & 25.5660 & 4211.1029 & 205.7544 & 4751.0444 \\
6 & 31.5277 & 6041.3146 & 211.8131 & 6581.2367 \\
7 & 41.3424 & 8207.2282 & 221.7421 & 8747.1272 \\
8 & 56.3963 & 10710.3116 & 236.9272 & 11250.1842 \\
9 & 78.2898 & 13552.2590 & 258.9689 & 14092.1016 \\
10 & 108.8385 & 16734.9903 & 289.6825 & 17247.7995 \\
\hline
\end{tabular}

\subsection{The case $\alpha<\beta_{n}^{2}$.}

In this case the solutions of the characteristic equation (37) will be

$$
\sqrt{\sqrt{\alpha}+\beta_{n}}, \quad-\sqrt{\sqrt{\alpha}+\beta_{n}}, \quad \sqrt{\beta_{n}-\sqrt{\alpha}}, \quad-\sqrt{\beta_{n}-\sqrt{\alpha}}
$$

and the solution of the differential equation (31) can be written in the form

$$
Y(y)=G_{1} \cosh \left(\sqrt{\sqrt{\alpha}+\beta_{n}} y\right)+G_{2} \sinh \left(\sqrt{\sqrt{\alpha}+\beta_{n}} y\right)+G_{3} \cosh \left(\sqrt{\beta_{n}-\sqrt{\alpha}} y\right)+G_{4} \sinh \left(\sqrt{\beta_{n}-\sqrt{\alpha}} y\right)
$$

where $G_{1}, G_{2}, G_{3}$, and $G_{4}$ are constants of integration.

By substituting (38) into the four boundary conditions (32)-(34) a system of four equations for $G_{1}$, $G_{2}, G_{3}$, and $G_{4}$ is obtained. To find nontrivial solutions for $Y(y)$ the determinant of the corresponding coeffitient matrix should be set equal to zero, that is,

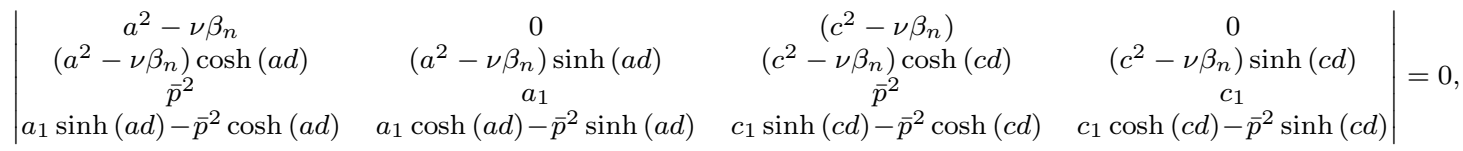

where $a=\sqrt{\sqrt{\alpha}+\beta_{n}}, c=\beta_{n}-\sqrt{\sqrt{\alpha}}, a_{1}=a\left(a^{2}-(2-\nu) \beta_{n}\right)$ and $c_{1}=c\left(c^{2}-(2-\nu) \beta_{n}\right)$. The eigenvalues $\alpha$ can be calculated from (41). Some numerical approximations of $\alpha$ are given in Table 3 for some values of the parameters.

Table 3.

\begin{tabular}{|r|l|l|l|l|l|l|}
\hline $\mathrm{n}$ & \multicolumn{9}{|c|}{$l=10, d=1, \bar{p}^{2}=1$} \\
\hline & $\nu=0.3$ & $\nu=0.4$ & $\nu=0.5$ & $\nu=0.6$ & \multicolumn{2}{|c|}{$\nu=0.8$} \\
\hline 5 & & & & & 5.0474 & \\
6 & & & 12.5524 & 11.4163 & 8.1418 & \\
7 & & 23.1493 & 21.6909 & 19.4993 & 13.3306 & \\
8 & & 38.1964 & 35.6143 & 32.1065 & 21.4984 & \\
9 & 62.9993 & 60.2307 & 56.2396 & 50.7482 & 33.7101 & \\
10 & 95.1879 & 91.1683 & 85.3165 & 77.1611 & 51.2067 & \\
11 & 138.7581 & 133.1580 & 124.9248 & 113.3053 & 75.3987 & 136.9501 \\
12 & 196.1310 & 188.5811 & 177.3735 & 161.3620 & 107.8584 & 178.9353 \\
13 & 269.9607 & 260.0502 & 245.1999 & 223.7315 & 150.3134 & 231.0134 \\
\hline
\end{tabular}


Extension of Table 3 .

\begin{tabular}{|r|l|l|l|l|l|l|}
\hline $\mathrm{n}$ & \multicolumn{7}{|c|}{$l=10, d=1, \bar{p}^{2}=1$} \\
\hline & $\nu=0.3$ & $\nu=0.4$ & $\nu=0.5$ & $\nu=0.6$ & \multicolumn{2}{c|}{$\nu=0.8$} \\
\hline 14 & 363.1342 & 350.4097 & 331.1689 & 303.0322 & 204.6407 & 294.8865 \\
15 & 478.7715 & 462.7357 & 438.2735 & 402.1001 & 272.8618 & 372.4121 \\
16 & 620.2261 & 600.3362 & 569.7342 & 523.9882 & 357.1389 & 465.6039 \\
17 & 791.0843 & 766.7508 & 729.9995 & 671.9661 & 459.7719 & 576.6301 \\
18 & 995.1661 & 965.7514 & 919.7457 & 849.5199 & 583.1959 & 707.8135 \\
19 & 1236.5247 & 1201.3419 & 1145.8771 & 1060.3514 & 729.9795 & 861.6300 \\
20 & 1519.4465 & 1477.7582 & 1411.5258 & 1308.3781 & 902.8236 & 1040.7077 \\
\hline
\end{tabular}

\subsection{The case $\alpha=\beta_{n}^{2}$.}

In this case the characteristic equation (37) becomes

$$
k^{2}\left(k^{2}-2 \beta_{n}\right)=0
$$

and its solutions are

$$
k_{1,2}=0, \quad k_{3,4}= \pm \sqrt{2 \beta_{n}} .
$$

The solution of the differential equation (31) is then given by

$$
Y(y)=S_{1}+S_{2} y+S_{3} \cosh \left(2 \sqrt{\beta_{n}}\right)+S_{4} \sinh \left(2 \sqrt{\beta_{n}}\right),
$$

where $S_{1}, S_{2}, S_{3}$, and $S_{4}$ are constants of integration. As in the previous two cases the following determinant is similary obtained when we look for nontrivial solutions of the boundary value problem for $Y(Y)$ (where $Y(y)$ is given by (43)): Like in previous two cases using boundary conditions (32)-(34) the system of the four equations for the determination of eigenvalues is recieved. This system has nontrivial solution when the determinant of the coefficient matrix for the unknown quantaties $S_{i}=0, i=1,2,3,4$ is equal to zero. In this case such determinant has following form:

$$
\left|\begin{array}{cccc}
-\nu \beta_{n} & 0 & (2-\nu) \beta_{n} & 0 \\
-\nu \beta_{n} & -\nu \beta_{n} d & (2-\nu) \beta_{n} \cosh \left(b_{1} d\right) & (2-\nu) \beta_{n} \sinh \left(b_{1} d\right) \\
\bar{p}^{2} & -(2-\nu) \beta_{n} & \bar{p}^{2} & \nu \beta_{n} b_{1} \\
-\bar{p}^{2} & -\left(\bar{p}^{2} d+(2-\nu) \beta_{n}\right) & \nu \beta_{n} b_{1} \sinh \left(b_{1} d\right)-\bar{p}^{2} \cosh \left(b_{1} d\right) & \nu \beta_{n} b_{1} \cosh \left(b_{1} d\right)-\bar{p}^{2} \sinh \left(b_{1} d\right)
\end{array}\right|=0,
$$

where $b_{1}=\sqrt{2 \beta_{n}}$. Solutions exist for some special values of the parameters. For example for $l=100, d=$ $0.1, \bar{p}^{2}=1, \nu=0.6$ solutions for $\alpha$ exist for first five modes. For $l=100, d=1, \bar{p}^{2}=1, \nu=0.6$ solutions exist for the first three modes and these solutions for $\alpha$ nwill be exactly the same las for $d=0.1$. This is due to the fact that $\alpha=\beta_{n}^{2}=\left(\frac{n \pi}{l}\right)^{4}$ and that $\alpha$ depends only on $n$ and $l$. The other parameters such as $\nu$ and $\bar{p}^{2}$ will only determine the existnce of nontrivil solutions $Y(y)$.

\section{Conclusions and remarks.}

In this paper the free vibrations of a rectangular plate with two opposite sides simply supported and the other two densely attached to linear springs have been studied. This combination of boundary conditions seems to be not considered in the literature before. This rectangular plate model is one of the simplest models to describe a suspension bridge. For the rectangular plate model the relationship between the plate parameters and the frequencies has been obtained by using an adapted version of the method of separation of variables (see [18]). This result is important to investigate the wind-induced oscillations of a rectangular plate. The relationship between the plate parameters and the frequencies has been obtained analytically. For some values of the parameters numerical approximations of the frequencies are given. 


\section{References}

[1] A.W. Leissa, Vibration of plates, NASA SP-160, 1969.

[2] A.W. Leissa, The free vibration of rectangular plates, J. of Sound and Vibration, Vol. 31, No.3 (1973), pp. 257-293.

[3] A.W. Leissa, P.A.A. Laura, R.H. Guiterrez,Vibrations of rectangular plates with nonuniform elastic edge supports, J. of Applied Mechanics, Vol 47 (1980) pp. 891-895.

[4] D.J. Gorman, Free vibration analysis of rectangular plates, Elsevier, New York, 1982.

[5] D.J. Gorman, Vibration analysis of plates by the superposition method, World Scientific, Singapore, 1999.

[6] T. Sakata and K. Hokosawa,Vibration of Clamped orthotropic rectangular plates, J. of Sound and Vibration, Vol. 125 (1988), pp. 439-439.

[7] P.A.A. Laura and R.O. Grossi,Transverse vibration of a rectangular plate elastically restrained against translation and rotation, J. of Sound and Vibration, Vol. 75 (1981), pp. 101-107.

[8] P.A.A. Laura, L.E. Luisoni and C.P. Filipich,A note on the determination of the fundamental frequency of vibration of thin rectangular plates with edges possessing different rotation flexibility coefficients, J. of Sound and Vibration, Vol. 155 (1997), pp. 327-333.

[9] W.L. Li and M. Daniels,A Fourier series method for the vibrations of elastically restrained plates arbitrarily loaded with springs and masses, J. of Sound and Vibration, Vol. 252, No. 4 (2002), pp. 768-781.

[10] A.C. Lazer, P.J. McKenna, Large-amplitude periodic oscillations in suspension bridges: Some new connections with nonlinear analysis, SIAM Rev., No. 32, (1990), pp. 537-578.

[11] P.J. McKenna, W.Walter, Traveling waves in a suspension bridge, SIAM J. Appl. Math., No.50 (1990), pp. 702-715.

[12] W.T. van Horssen, An asymptotic theory for a class of initial-boundary value problems for weakly nonlinear wave equations with an application to a model of the galloping oscillations of overhead transmission lines, SIAM J. Appl. Math., No. 48 (1988), pp. 1227-1243.

[13] G.J. Boertjens, W.T. van Horssen, On mode interactions for a weakly nonlinear beam equation, Nonlinear Dynam., No. 17 (1998), pp. 23-40.

[14] G.J. Boertjens, W.T. van Horssen, An asymptotic theory for a weakly nonlinear beam equation with a quadratic perturbation, SIAM J. Appl. Math.,Vol. 60, No. 2 (2000), pp. 602-632.

[15] Q.H. Choi, T. Jung, On periodic solutions for the nonlinear suspension bridge equation, Differential Integral Equations, No. 4 (1991), pp. 383-396.

[16] G. Tajčovâa,Mathematical models of suspension bridges, Applications of Mathematics, Vol. 42, No. 6 (1997), pp. 451-480

[17] W.T. van Horssen, M.A. Zarubinskaya, On an elastic dissipation model for a cantilevered beam, (to appear in Quarterly of Applied Mathematics (2003)).

[18] W.T. van Horssen, On the applicability of the method of separation of variables for partial difference equations, J. of Difference Equations and Applications, Vol. 8 (1), (2002), pp.53-60. 